\title{
Brolucizumab in Neovascular Age-Related Macular Degeneration - Indian Real-World Experience: The BRAILLE Study
}

\author{
Debdulal Chakraborty ${ }^{\prime}$ \\ Aniruddha Maiti ${ }^{2}$ \\ Jay U Sheth (D) $^{3}$ \\ Subhendu Boral' \\ Soumen Mondal' \\ Krishnendu Nandi ${ }^{2}$ \\ Tushar Sinha' \\ Arnab Das' \\ 'Department of Vitreoretinal Services, \\ Disha Eye Hospitals, Kolkata, West \\ Bengal, India; ${ }^{2}$ Department of \\ Vitreoretinal Services, Netralayam Super \\ Speciality Eye Care Centre, Kolkata, \\ West Bengal, India; ${ }^{3}$ Department of \\ Vitreoretinal Services, Surya Eye Institute \\ and Research Center, Mumbai, \\ Maharashtra, India
}

Purpose: To assess the short-term efficacy and safety profile of intravitreal brolucizumab injection in Indian eyes with neovascular age-related macular degeneration (nAMD) under real-world conditions.

Patients and Methods: This was a multicenter, retrospective chart review of 94 eyes of 94 patients with nAMD (treatment-naïve and switch-therapy) undergoing brolucizumab therapy. Re-treatment as per pro-re-nata protocol was performed based on fixed visual and tomographic criteria. The main outcome measures were changes in the best-corrected visual acuity (BCVA), intraretinal fluid (IRF), subretinal fluid (SRF), central subfield thickness (CST), and pigment epithelial detachment (PED) along with safety analysis.

Results: Of the 94 eyes, 20 eyes (21.3\%) were treatment-naïve, whereas the rest 74 eyes $(78.7 \%)$ underwent switch therapy. One hundred and twenty-six injections were given over a mean followup of $7.3 \pm 2.2$ (range $5-30$ ) weeks. The BCVA improved significantly from $0.82 \pm 0.5$ LogMAR at baseline to $0.66 \pm 0.5 \operatorname{LogMAR}$ at the final visit $(\mathrm{p}<0.0001)$. Significant reduction in CST was simultaneously noted (Baseline: $408.45 \pm 65.63 \mu \mathrm{m}$; Final: $281.14 \pm 37.74 \mu \mathrm{m}$; $\mathrm{p}<0.0001$ ). On qualitative analysis, resolution of subretinal fluid (SRF), intraretinal fluid (IRF), and pigment epithelial detachment (PED) was observed in $15.5 \%, 39.29 \%$, and $23.81 \%$ of the eyes, respectively. The mean interval of repeat injection was $10.2 \pm 2.1$ weeks. Three episodes of ocular adverse drug reaction were reported, including two patients developing subretinal hemorrhage while one having a retinal pigment epithelial (RPE) tear. Notably, no intraocular inflammation (IOI) was seen in any of the eyes, and no systemic side effects were identified.

Conclusion: In a real-world scenario, brolucizumab therapy is efficacious and safe in the management of nAMD over the short term. Further long-term studies are warranted to validate these findings. Additionally, lack of ocular inflammation after 126 brolucizumab injections in our Indian data is peculiar and underlines the necessity to explore the role of race and genetics in predisposing to/safeguarding against brolucizumab-related IOIs.

Keywords: brolucizumab, inflammation, age-related macular degeneration

\section{Introduction}

Age-related macular degeneration (AMD) is a chronic, progressive disease of the central retina and a major cause of irreversible vision loss worldwide. ${ }^{1}$ Biologics that inhibit the angiogenic action of vascular endothelial growth factor (anti-VEGF) has revolutionized the treatment of the neovascular form of AMD (nAMD). ${ }^{2}$ These anti-VEGF agents need to be administered frequently for optimal outcomes in the management of nAMD. The economics and logistics of repeated injections, on the
Correspondence: Debdulal Chakraborty Department of Vitreoretinal Services, Disha Eye Hospitals, Kolkata, West Bengal, India

Tel +9l 9433059923

Email devdc.dr@gmail.com 
other hand, remain a challenge. As a result, many patients in real-world settings do not receive treatment as frequently as they do in standardized clinical trials, resulting in poor visual outcomes. ${ }^{3}$

The anti-VEGF agents which are currently being used include bevacizumab (Avastin ${ }^{\circledR}$; Genentech, S. San Francisco, CA/Roche, Basel, Switzerland), ranibizumab (Lucentis ${ }^{\circledR}$; Genentech, S. San Francisco, CA/Roche, Basel, Switzerland), aflibercept $\left(\right.$ Eylea $^{\circledR}$, Regeneron, Tarrytown, NY), and brolucizumab (Beovu ${ }^{\circledR}$; Novartis, Basel, Switzerland). ${ }^{4-6}$ Of these, brolucizumab is the latest agent to be approved by the United States Food and Drug Administration (US FDA) for the treatment of nAMD. In India, the drug was recently approved by the Drug Controller General of India (DCGI) and was marketed and used as Pagenax ${ }^{\circledR}$ (Novartis India Ltd, Mumbai, India) since October 2020. The HAWK and the HARRIER studies were pivotal trials demonstrating its efficacy and safety in nAMD. ${ }^{7}$ These trials provide an ideal platform for formulating guidelines for the management of nAMD due to their methodology, strict treatment regimen, and delivery settings. The results obtained in these controlled settings, however, are rarely duplicated in real-world scenarios.

The real-world clinical data of intravitreal injection (IVI) of brolucizumab in the management of nAMD in Indian eyes has not been reported. To address this gap in the literature, we describe the early visual acuity and tomographic outcomes of IVI brolucizumab in treatmentnaïve and previously treated eyes with nAMD. Additionally, we seek to evaluate the safety profile of IVI brolucizumab in Indian eyes.

\section{Patients and Methods}

This was a multi-center, retrospective consecutive, interventional, uncontrolled, nonrandomized study incorporating data from four tertiary care centers in India. The study was conducted in accordance with the tenets of the Declaration of Helsinki and was approved by the Institutional Review Board at Disha Eye Hospitals (Kolkata, India), Susrut Eye Hospital (Kolkata, India), B B Eye Foundation (Kolkata, India) and Surya Eye Institute and Research Center (Mumbai, India). Written informed consent for treatment and data collection was obtained from each patient.

\section{Design}

The electronic database search was performed for nAMD patients who underwent IVI brolucizumab therapy between October 2020 and February 2021. All consecutive patients who were either treatment-naïve or those who had previously received repeated anti-VEGF therapy with either ranibizumab or aflibercept for recalcitrant fluid were included in the study. Recalcitrant fluid was defined as persistent fluid ( $<100 \mu \mathrm{m}$ reduction in fluid) or worsening of fluid on spectral-domain optical coherence tomography (SD-OCT). The diagnosis of nAMD was based on a combination of clinical findings, fundus fluorescein angiography (FFA), and indocyanine green angiography (ICGA) to rule out polypoidal choroidal vasculopathy (PCV). On FFA, nAMD was defined as areas of stippled hyperfluorescence with progressive leakage or late leakage of undermined source or presence of early well-defined hyperfluorescence network with progressive leakage in late phase. The exclusion criteria included eyes with coexisting vitreoretinal pathology other than nAMD, choroidal neovascularization (CNV) due to any other etiology, significant media opacities that precluded the observation of the ocular fundus, past history of any retinal surgery, coexisting diabetic retinopathy, any history of systemic vasculitis or autoimmune disease or any history of anterior or posterior segment inflammation. For treatment-naïve nAMD patients, choices of all anti-VEGF agents were offered, and patients freely chose to receive IVI brolucizumab (6 $\mathrm{mg}$ in $0.05 \mathrm{~mL}$ ).

All injections were performed in an operating theater under sterile technique. Topical moxifloxacin $0.5 \%$ was administered postoperatively for one week. The patients were reviewed on day 1 , and at week 1,2 , and 4 , respectively, and every four weeks thereafter. Additionally, the patients were advised to follow up immediately in case of occurrence of any ocular or systemic adverse event. At baseline and all subsequent follow-up visits, the patients underwent a detailed clinical examination including bestcorrected visual acuity (BCVA) assessment using the Snellen's visual acuity chart, intraocular pressure (IOP) measurement by Goldmann applanation tonometer, anterior segment evaluation using slit-lamp biomicroscopy and fundus examination with both slit-lamp biomicroscopy (+90D lens) and indirect ophthalmoscopy (+20D lens) and SD-OCT. A detailed history regarding the occurrence of any ocular or systemic adverse event was taken at each visit. Retreatment was offered to all the patients if there was persistent fluid ( $<100 \mu \mathrm{m}$ reduction in fluid) or worsening of fluid or drop of $\geq$ one Snellen's line visual acuity, based on pro-re-nata (PRN) regimen. All the 
demographic and clinical data were extracted from the electronic medical database.

\section{Outcome Measures}

Primary outcome measures included the change in BCVA and improvement in central subfield thickness (CST) from the baseline along with safety analysis. The secondary outcomes measures were changes in the subretinal fluid (SRF), intraretinal fluid (IRF), and pigment epithelial detachment (PED). Only fibrovascular PED, defined as irregular retinal pigment epithelial (RPE) elevation along with a non-uniform interior comprising material with variable reflectivity, was evaluated. In the presence of multiple PEDs, the PED with the largest area was considered for analysis. All imaging analysis was performed by two independent graders (D.C., A.M.). In case of any discrepancies, the image was analyzed together by the graders and a consensus achieved.

A detailed evaluation of the ocular and systemic safety profile was performed as described by our group earlier. ${ }^{8}$ Briefly, the adverse events (AEs) and adverse drug reactions (ADRs) were identified in the medical records. Based on the "Harmonised Tripartite Guidelines on Clinical Safety Data Management (E2A)" by the "International Council for Harmonisation of Technical Requirements for Pharmaceuticals for Human Use (ICH)", an AE is defined as Any untoward medical occurrence in a patient or clinical investigation subject administered a pharmaceutical product and which does not necessarily have to have a causal relationship with this treatment.

Similarly, "All noxious and unintended responses to a medicinal product related to any dose should be considered ADR", whereby The phrase 'responses to a medicinal products' means that a causal relationship between a medicinal product and an adverse event is at least a reasonable possibility, i.e., the relationship cannot be ruled out. ${ }^{9}$

The AEs and ADRs were sub-classified as serious AEs (SAE) or serious ADRs (sADR), and non-serious AEs (nsAE) or non-serious ADRs (nsADR). An SAE or sADR was defined as any event that occurred at any dose that resulted in death, a life-threatening $\mathrm{AE}$, required inpatient hospitalization or prolongation of existing hospitalization, resulted in persistent or significant disability/ incapacity, or a congenital anomaly/birth defect. Events that did not fulfill these criteria were labeled as nsAE or nsADR. All AEs were reported by the treating vitreoretinal specialist and categorized based on his/her clinical judgment.

\section{Statistical Analysis}

The statistical analysis was performed by SPSS 23.0 version (SPSS Inc., Chicago, Ill., USA). Continuous variables were described as mean and variation of each observation from the mean value (Standard deviation) represented as mean \pm standard deviation (SD) or median (Interquartile range; IQR) if they failed to follow a normal distribution. Paired data in comparison with baseline were analyzed with paired $t$-test (normal distribution) or Wilcoxon signed-rank test (for non-normal distribution). Categorical variables were described by taking percentages; paired data in comparison with baseline was analyzed using the McNemar test. Variables with a p-value $<$ 0.05 were considered statistically significant.

\section{Results}

A total of 94 eyes of 94 patients were included in the study. Of these, 20 eyes $(21.3 \%)$ were treatment-naïve whereas the remaining 74 eyes (78.7\%) underwent switch therapy. The mean age of patients was $67.55 \pm 10.25$ years with a majority being males (63 patients: $67.7 \%$ ). The mean follow-up period was $7.3 \pm 2.2$ (range 5-30) weeks after the first injection of IVI brolucizumab. The patients received a total of 126 injections with a mean of $1.36( \pm$ $0.58)$. Of these, 65 eyes $(69.1 \%)$ received a single injection, 24 eyes $(25.53 \%)$ received two injections, while 5 eyes $(5.31 \%)$ received three IVI brolucizumab. Patients who underwent more than one injection of IVI brolucizumab (29/94 eyes; $30.85 \%)$ received it at an interval of 10.2 \pm 2.1 weeks. The mean number of previous anti-VEGF injections in the switch group before administering IVI brolucizumab $8.63 \pm 4.74$ (range 3-44). Table 1 provides the descriptive analysis of the cohort.

\section{Visual Acuity}

Significant improvement in BCVA from baseline to final visit was noted in the eyes after IVI brolucizumab (Baseline LogMAR BCVA: $0.81 \pm 0.5$; Final LogMAR BCVA: $0.66 \pm$ $0.51 ; \mathrm{p}<0.00001)$. On subgroup analysis, although an improvement in BCVA was noted in the treatment-naïve group (Baseline LogMAR BCVA: $0.41 \pm 035$; Final LogMAR BCVA: $0.36 \pm 0.41 ; p=0.36$ ), it was not statistically significant. In contrast, the switch group demonstrated a significant improvement in the final BCVA (Baseline LogMAR BCVA: $0.91 \pm 049$; Final LogMAR BCVA: 0.73 $\pm 0.51 ; p<0.00001)$. The values have been summarized in Table 2. 
Table I Demographic Characteristics of the Study Population

\begin{tabular}{|l|c|}
\hline Characteristic & $\begin{array}{c}\text { Number of Patients } \\
\text { (Total 94) }\end{array}$ \\
\hline $\begin{array}{l}\text { Age (years) } \\
\text { Mean }( \pm S D)\end{array}$ & $67.25(10.25)$ \\
\hline $\begin{array}{l}\text { Gender } \\
\text { Males }\end{array}$ & $63(67.7 \%)$ \\
Females & $31(32.3 \%)$ \\
\hline $\begin{array}{l}\text { Treatment Status } \\
\text { Treatment-naive }\end{array}$ & $20(21.3 \%)$ \\
Previously treated & $74(78.7 \%)$ \\
\hline $\begin{array}{l}\text { Total number of prior injections in switch } \\
\text { therapy group } \\
\text { Mean }( \pm S D)\end{array}$ & $8.63(4.74)$ \\
\hline $\begin{array}{l}\text { Total number of IVI brolucizumab per } \\
\text { patient } \\
\text { Mean }( \pm S D)\end{array}$ & $1.36( \pm 0.58)$ \\
\hline $\begin{array}{l}\text { Interval between IVI brolucizumab in } \\
\text { weeks } \\
\text { Mean }( \pm S D)\end{array}$ & $10.2 \pm 2.1$ \\
\hline
\end{tabular}

Abbreviations: SD, standard deviation; IVI, intravitreal injection.

\section{SDOCT Analysis}

The overall mean central subfoveal thickness (CST) at the baseline was $408.45 \pm 65.63 \mu \mathrm{m}$, which improved significantly to $281.14 \pm 37.74 \mu \mathrm{m}(\mathrm{P}<0.0001)$ (Figure 1$)$. On subgroup analysis, a significant reduction in CST was noted in both the treatment-naive and the switch therapy groups (Treatment-naïve eyes group: Baseline CST- $398.53 \pm 61.38$ $\mu \mathrm{m}$; Final CST- $285.16 \pm 15.14 \mu \mathrm{m} ; \mathrm{p}<0.0001$; Switch therapy group: Baseline CST- $410.96 \pm 66.42 \mu \mathrm{m}$; Final CST- $280.12 \pm 41.5 \mu \mathrm{m} ; \mathrm{p}<0.0001)$. The values have been summarized in Table 2. Out of the 71 eyes with SRF at baseline, complete resolution was seen in 11 eyes $(15.49 \%)$, reduced in 56 eyes $(78.87 \%)$ and minimal/no change was noted in 4 eyes $(5.63 \%)$. Among the 84 eyes with IRF at baseline, 33 eyes (39.29\%) showed a complete resolution of IRF, while it was reduced in 39 eyes $(46.43 \%)$ and showed minimal/no change in 12 eyes (14.29\%). PED was seen in 21 eyes $(22.34 \%)$ at baseline, which was resolved in 5 eyes (23.81\%) reduced in 13 eyes $(61.9 \%)$ and in 3 eyes (14.29\%) persisted without any change. Figures 1 and 2 are representative case examples of treatment-naïve and previously treated cases of nAMD undergoing IVI brolucizumab.

\section{Safety Analysis}

Adverse events were noted after 31 (24.6\%) IVI brolucizumab injections; of these AEs, 28 were nsAE (90.32\%) and 3 were ocular sADR (9.68\%). The non-serious AEs occurred with $22.22 \%$ of all injections, with mild ocular pain $(14.29 \%$ of the total number of injections), burning sensation (4.76\% of the total number of injections), and subconjunctival hemorrhage $(3.17 \%$ of the total number of injections) being most common. Serious ocular ADR occurred after $2.38 \%$ ( 3 eyes) of all the injections. Two eyes $(1.59 \%$ of the total number of injections) developed subretinal hemorrhage for which a vitrectomy was performed. Retinal pigment epithelial tear developed in one eye $(0.79 \%$ of the total number of injections). No nsADR, including any episode of raised IOP, or any systemic sADR such as systemic thromboembolic event was reported in the study. No cases of intraocular inflammation (IOI) were identified. The values have been summarized in Table 3 .

\section{Discussion}

In this real-world study on IVI brolucizumab therapy in nAMD, a significant improvement in visual acuity along with a notable reduction in the CST was observed. A detailed tomographic evaluation demonstrated a reduction in the amount of fluid, both intra- and subretinally, and PED dimensions. Adverse events were noted in $24.6 \%$ of injections, but most $(90.32 \%)$ were non-

Table 2 Differences in the Pre- and Post-Intravitreal Brolucizumab Injection Variables

\begin{tabular}{|c|c|c|c|c|c|}
\hline \multicolumn{3}{|c|}{ Characteristic } & Preinjection & Postinjection & P-value \\
\hline LogMAR BCVA & $\begin{array}{l}\text { Entire cohort } \\
\text { Treatment-naïve } \\
\text { Switch Therapy }\end{array}$ & Mean $( \pm \mathrm{SD})$ & $\begin{array}{l}0.8 \mathrm{I}(0.5) \\
0.4 \mathrm{I}(0.35) \\
0.9 \mathrm{I}(0.49)\end{array}$ & $\begin{array}{l}0.66(0.5 \mathrm{I}) \\
0.36(0.4 \mathrm{I}) \\
0.73(0.5 \mathrm{I})\end{array}$ & $\begin{array}{c}<0.00001 \\
0.36 \\
<0.00001\end{array}$ \\
\hline CST & $\begin{array}{l}\text { Entire cohort } \\
\text { Treatment-naïve } \\
\text { Switch Therapy }\end{array}$ & Mean $( \pm S D)$ & $\begin{array}{l}408.45(65.63) \\
398.53(61.38) \\
410.96(66.42)\end{array}$ & $\begin{array}{c}281.14(37.74) \\
285.16(15.14) \\
280.12(41.5)\end{array}$ & $\begin{array}{l}<0.00001 \\
<0.00001 \\
<0.00001\end{array}$ \\
\hline
\end{tabular}

Abbreviations: LogMAR, Logarithm of the Minimum Angle of Resolution; BCVA, Best-corrected visual acuity; SD, Standard deviation; CST, Central subfield thickness. 

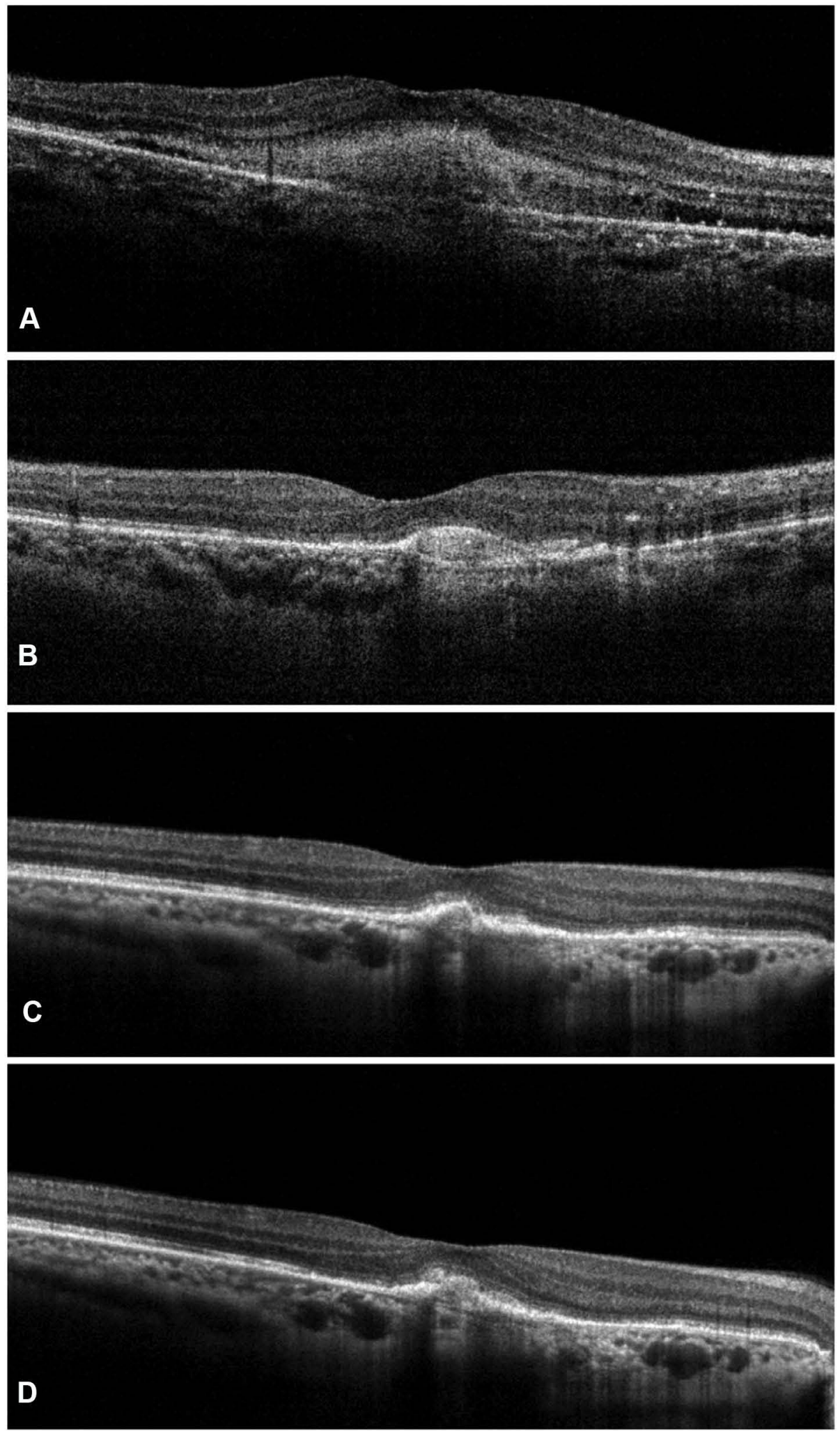

Figure I Representative case of a treatment-naïve nAMD showing complete resolution of subretinal fluid along with significant reduction in the subretinal hyperreflective material (SHRM) at weeks 4 (B), 8 (C), and 12 (D) as compared to the baseline (A) after a single dose of intravitreal brolucizumab injection. 

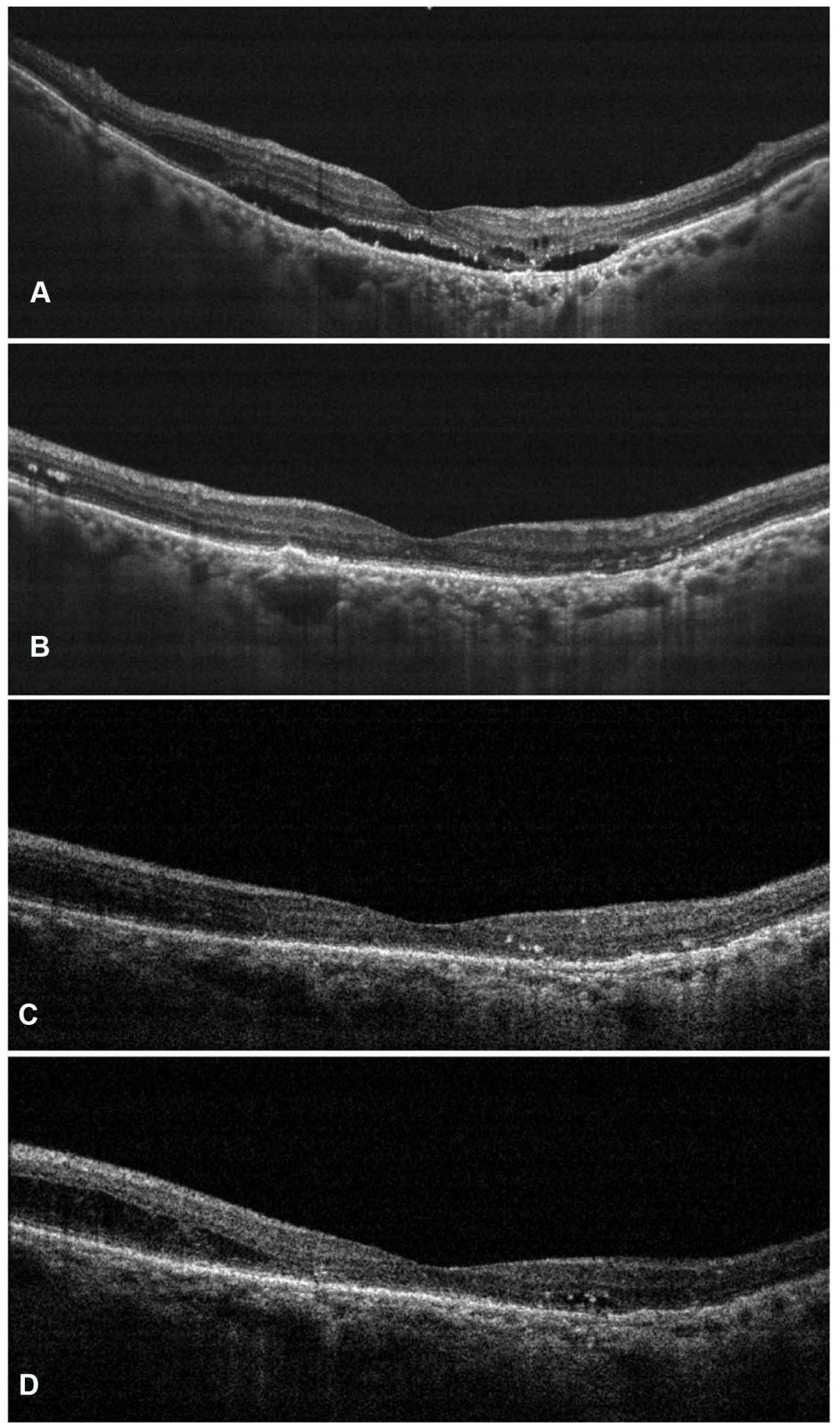

Figure 2 Representative case of nAMD who had previously received multiple anti-vascular endothelial growth factor (anti-VEGF) injections with suboptimal outcomes. After switching to intravitreal injection (IVI) brolucizumab, the patient demonstrated complete resolution of the subretinal and intraretinal fluid at weeks 4 (B) and 8 (C) as compared to the baseline (A). Early recurrence was noted at I2 weeks (D) for which the patient underwent second dose of IVI brolucizumab. 
Table 3 List of Adverse Events in Patients Receiving Intravitreal Brolucizumab Injections

\begin{tabular}{|l|c|}
\hline Adverse Event & $\begin{array}{c}\text { Frequency } \\
\text { (\%) }\end{array}$ \\
\hline Total & $31(24.6)$ \\
(Number of events/Total number of injections & \\
$[126])$ & \\
\hline nsAE & \\
(Number of events/Total number of injections & \\
[I26]) & $18(14.29 \%)$ \\
Mild ocular Pain & $6(4.76)$ \\
Burning sensation & $4(3.17)$ \\
Subconjunctival hemorrhage & $28(22.22)$ \\
Total & \\
\hline sADR (Ocular) & \\
(Number of events/Total number of injections & $2(1.59)$ \\
[I26]) & I (0.79) \\
Subretinal hemorrhage & $3(2.38)$ \\
RPE tears & \\
Total & \\
\hline
\end{tabular}

Abbreviations: nsAE, Non-serious adverse events; sADR, Serious adverse drug reaction; RPE, Retinal pigment epithelium.

serious. Interestingly, no cases of IOI were seen after 126 IVI brolucizumab.

Anti-VEGF therapy has become the standard of care for the management of macular neovascularization $(\mathrm{MNV}){ }^{2}$ Each anti-VEGF agent which is currently in use offers certain unique advantages. While bevacizumab is the most economical choice, ranibizumab has been FDA-approved for the past 14 years and has the most evidence in the literature. Aflibercept acts against multiple molecular targets including VEGF-A, VEGF-B, and placental growth factor $[\mathrm{PlGF}]$ and has the advantage of a bi-monthly dosing schedule. ${ }^{2,10}$ Brolucizumab is a very durable agent primarily due to its low molecular weight allowing for higher molar dosing and offers the advantage of $\geq 12$ weekly dosage in nAMD. ${ }^{10-12}$ We used a PRN regimen in the current cohort because of the patient population's socioeconomic profile and affordability. We noted a mean time to the next injection of $10.2 \pm 2.1$ weeks, which is in accordance with the recommended quarterly regimen after the initial three loading doses.

The HAWK and the HARRIER trials demonstrated the non-inferiority of IVI brolucizumab to aflibercept in visual outcomes at 96 -weeks. ${ }^{7}$ In these trials, the final visual improvement noted with $6 \mathrm{mg}$ brolucizumab was a modest 5.9 and 6.1 ETDRS letters, respectively. While the HAWK and HARRIER cohorts had a mean baseline VA of 60.6 (HAWK) and 61.2 (HARRIER) ETDRS letters (corresponding nearly to $0.47-0.49 \log M A R$ ), in our realworld retrospective cohort the mean baseline VA was comparatively worse at 0.81 LogMAR. Despite the fact that the current study's participants had poor baseline vision, considerable visual improvement was observed at the last visit. These visual benefits were sustained after stratification in the eyes that had been switched from other anti-VEGF medications. In contrast, the SHIFT and BREW studies, which looked at real-world data on brolucizumab after switching, found no significant visual improvements. ${ }^{13,14}$ In a comparable study, Enriquez et al found no significant visual improvement in 166 eyes undergoing switch therapy and 6 treatment-naive eyes with nAMD. ${ }^{15}$ The lack of significant improvement in BCVA among the treatment-naive eyes was intriguing to note in our investigation, despite a positive trend toward visual gains. In contrast, Bilgic et al observed that brolucizumab improved vision in both treatment-naive and switch therapy eyes with nAMD. ${ }^{16}$ The "ceiling effect" in patients with very good visual acuity at baseline can also account for the varying visual gains between studies. ${ }^{17}$ This can explain the lack of significant visual gain in the treatment-naïve group of our study, who had better mean visual acuity at baseline $(0.41[ \pm 0.35]$ LogMAR) as compared to the switch therapy eyes $(0.81$ $[ \pm 0.5]$ LogMAR).

Our real-world study demonstrates a definite trend towards an improvement amongst all SD-OCT biomarkers after brolucizumab. Both the treatment-naive and switch therapy groups showed a significant reduction in CST. The SHIFT trial, as well as Enriquez et al, have shown similar improvements in CST. ${ }^{13,15}$ Complete resolutions of SRF, IRF, and PED were observed in $15.5 \%, 39.29 \%$, and $23.81 \%$ of our study eyes. In comparison, the SRF resolution in the BREW trial was superior (39.4\%), while the PED resolution was suboptimal $(6.4 \%) .{ }^{16}$ At the same time, the IRF resolution in their eyes $(36.8 \%)$ was similar to the current study. ${ }^{16}$

Intraocular inflammation has been reported with other antiVEGFs in the past, at an estimated rate of between $0.3 \%$ and $2.9 \%$ per injection. ${ }^{18,19}$ The Fight Retinal Blindness! (FRB!) registry evaluated the incidence of infectious and noninfectious endophthalmitis in 88,150 intravitreal injections. ${ }^{20}$ They reported a higher rate of non-infectious endophthalmitis with bevacizumab $(8 / 9931 ; 0.081 \%)$ as compared to 
ranibizumab (3/54,776, 0.005\%; $\mathrm{P}=0.005)$ and aflibercept $(0 /$ 23,425; $\mathrm{P}=0.016$ ). In the HAWK and the HARRIER trials, the incidence of IOI was $4 \%$ with brolucizumab as compared to $1 \%$ with aflibercept. ${ }^{7}$ In the real-world, the SWIFT trial found that $12.4 \%$ of 207 IVI brolucizumab patients had IOI, while Enriquez et al found that $8.1 \%$ of the 172 eyes studied had IOI. ${ }^{15}$ In our series of 126 brolucizumab injections, however, we found no cases of IOI. The etiopathogenesis of brolucizumab-induced inflammation remains undetermined, although efforts are currently underway to explore the same. An interesting observation is that although IOI has been reported with other anti-VEGF agents, the episodes are fewer and are largely confined to mild to moderate anterior segment inflammation and vitritis. In contrast, the usage of brolucizumab has been associated with visual threatening occlusive vasculitis. ${ }^{15,21}$ In fact, the American Society of Retina Specialists (ASRS) released an alert on February 23 and March 30, 2020, inflammatory issues with the brolucizumab molecule. ${ }^{22}$ In the post-marketing surveillance, the incidence of retinal vasculitis +/retinal vascular occlusion has been 15.4 per 10,000 injections (till July 8, 2021). ${ }^{23}$ The fact that these inflammatory events were observed in the Caucasian population is important to highlight from the clinical trials and real-world data. ${ }^{15,21}$ Our real-world study, on the other hand, assessed the safety and efficacy in an Indian population, ie, Southeast Asians. Racial and genetic differences in the occurrence of systemic and ocular inflammation have been widely reported. ${ }^{24-29}$ The Pacific Ocular Inflammation Study found that among a group of 217,061 people, whites have a higher prevalence of ocular inflammation than nonwhites. ${ }^{26}$ Asians, on the other hand, had the highest prevalence of ocular inflammatory and infectious illnesses among 17,361,000 patients in the United States, according to the Intelligent Research in Sight (IRIS) registry. ${ }^{27}$ Thus, race and genetics may play a role in inflammatory response variability, and based on our preliminary results, this association needs to be investigated further pertaining to brolucizumab-induced IOI.

The major limitations of the present study are its retrospective design, limited sample size, brief follow-up, and the use of the PRN regimen. The study was also not powered for a safety analysis, which is consistent with previously published anti-VEGF injection safety data. ${ }^{30}$ Furthermore, because the study is based on medical chart analysis, there is a risk of adverse events, particularly mild ones, being under-reported. Apart from the racial and genetic differences, another likely possibility is the small sample size, which is insufficient to identify the relatively rare prevalence rate (15.4 per 10,000 injections) of vasculitis and/or occlusion. Despite these limitations, the results reported here represent the first real-world data regarding the efficacy and safety of brolucizumab in Indian eyes with nAMD.

\section{Conclusion}

In conclusion, the early real-world evidence suggests that IVI brolucizumab is safe and efficacious in the treatment of nAMD. Long-term prospective studies with a larger sample size and comparative arms are needed to validate our short-term results. Furthermore, including patients from various ethnic backgrounds with a diverse genetic pool is critical in determining their putative role in mediating brolucizumab-induced IOI.

\section{Disclosure}

The authors report no conflicts of interest in this work.

\section{References}

1. Stewart MW. Clinical and differential utility of VEGF inhibitors in wet age-related macular degeneration: focus on aflibercept. Clin Ophthalmol. 2012;6:1175-1186. doi:10.2147/OPTH.S33372

2. Finger RP, Guymer RH, Gillies MC, Keeffe JE. The impact of anti-vascular endothelial growth factor treatment on quality of life in neovascular age-related macular degeneration. Ophthalmology. 2014;121(6):1246-1251. doi:10.1016/j.ophtha.2013.12.032

3. Rao P, Lum F, Wood K, et al. Real-world vision in age-related macular degeneration patients treated with single anti-VEGF drug Type for 1 Year in the IRIS registry. Ophthalmology. 2018;125 (4):522-528. doi:10.1016/j.ophtha.2017.10.010

4. Li E, Donati S, Lindsley KB, Krzystolik MG, Virgili G. Treatment regimens for administration of anti-vascular endothelial growth factor agents for neovascular age-related macular degeneration. Cochrane Database Syst Rev. 2020;5(5):CD012208. doi:10.1002/14651858.CD012208

5. Campa C, Alivernini G, Bolletta E, Parodi MB, Perri P. Anti-VEGF therapy for retinal vein occlusions. Curr Drug Targets. 2016;17 (3):328-336. doi:10.2174/1573399811666150615151324

6. Mansour SE, Browning DJ, Wong K, Flynn HW Jr, Bhavsar AR. The evolving treatment of diabetic retinopathy. Clin Ophthalmol. 2020;14:653-678. doi:10.2147/OPTH.S236637

7. Dugel PU, Singh RP, Koh A, et al. HAWK and HARRIER: ninety-six-week outcomes from the Phase 3 trials of brolucizumab for neovascular age-related macular degeneration. Ophthalmology. 2021;128(1):89-99. doi:10.1016/j.ophtha.2020.06.028

8. Chakraborty D, Stewart MW, Sheth JU, et al. Real-world safety outcomes of intravitreal ranibizumab biosimilar (razumab) therapy for chorioretinal diseases. Ophthalmol Ther. 2021;10(2):337-348. doi:10.1007/s40123-021-00345-2

9. European Medicines Agency. ICH Topic E 2 A Clinical Safety Data Management: Definitions and Standards for Expedited Reporting; 1995. Available from: https://www.ema.europa.eu/en/documents/scientificguideline/international-conference-harmonisation-technical-require ments-registration-pharmaceuticals-human-use_en-15.pdf. Accessed July 9, 2021.

10. Garweg JG, Gerhardt C. Disease stability and extended dosing under anti-VEGF treatment of exudative age-related macular degeneration (AMD) - a meta-analysis. Graefes Arch Clin Exp Ophthalmol. 2021;259(8):2181-2192. doi:10.1007/s00417-020-05048-1 
11. Dugel PU, Jaffe GJ, Sallstig P, et al. Brolucizumab versus aflibercept in participants with neovascular age-related macular degeneration: a randomized trial. Ophthalmology. 2017;124(9):1296-1304. doi:10.1016/j.ophtha.2017.03.057

12. Dugel PU, Koh A, Ogura Y, et al. HAWK and HARRIER: Phase 3, multicenter, randomized, double-masked trials of brolucizumab for neovascular age-related macular degeneration. Ophthalmology. 2020;127(1):72-84. doi:10.1016/j.ophtha.2019.04.017

13. Bulirsch LM, Saßmannshausen M, Nadal J, Liegl R, Thiele S, Holz FG. Short-term real-world outcomes following intravitreal brolucizumab for neovascular AMD: SHIFT study. $\mathrm{Br} J$ Ophthalmol. 2021;bjophthalmol-2020-318672. doi:10.1136/bjophthalmol-2020318672

14. Sharma A, Kumar N, Parachuri N, et al. Brolucizumab-early real-world experience: BREW study. Eye (Lond). 2021;35 (4):1045-1047. doi:10.1038/s41433-020-1111-x

15. Enríquez AB, Baumal CR, Crane AM, et al. Early experience with brolucizumab treatment of neovascular age-related macular degeneration. JAMA Ophthalmol. 2021;139(4):441-448. doi:10.1001/jamaophthalmol.2020.7085

16. Bilgic A, Kodjikian L, March de Ribot F, et al. Real-world experience with brolucizumab in wet age-related macular degeneration: the REBA study. J Clin Med. 2021;10(13):2758. doi:10.3390/ jcm 10132758

17. Writing Committee for the UK Age-Related Macular Degeneration EMR Users Group. The neovascular age-related macular degeneration database: multicenter study of 92976 ranibizumab injections: report 1: visual acuity. Ophthalmology. 2014;121(5):1092-1101. doi:10.1016/j.ophtha.2013.11.031

18. Agrawal S, Joshi M, Christoforidis JB. Vitreous inflammation associated with intravitreal anti-VEGF pharmacotherapy. Mediators Inflamm. 2013;2013:943409. doi:10.1155/2013/943409

19. Johnson D, Sharma S. Ocular and systemic safety of bevacizumab and ranibizumab in patients with neovascular age-related macular degeneration. Curr Opin Ophthalmol. 2013;24(3):205-212. doi:10.1097/ICU.0b013e32835f8ec0

20. Daien V, Nguyen V, Essex RW, et al. Incidence and outcomes of infectious and noninfectious endophthalmitis after intravitreal injections for age-related macular degeneration. Ophthalmology. 2018;125 (1):66-74. doi:10.1016/j.ophtha.2017.07.005
21. Baumal CR, Spaide RF, Vajzovic L, et al. Retinal vasculitis and intraocular inflammation after intravitreal injection of brolucizumab. Ophthalmology. 2020;127(10):1345-1359. doi:10.1016/j. ophtha.2020.04.017

22. Beovuß. Post-marketing data in patients with wet AMD; 2021. Available from: https://www.brolucizumab.info/post-marketing-data. Accessed July 9, 2021.

23. Sharma A, Kumar N, Parachuri N, et al. Brolucizumab and immunogenicity. Eye (Lond). 2020;34(10):1726-1728. doi:10.1038/ s41433-020-0853-9

24. Paalani M, Lee JW, Haddad E, Tonstad S. Determinants of inflammatory markers in a bi-ethnic population. Ethn Dis. 2011;21 (2):142-149

25. Angeles-Han ST, McCracken C, Yeh S, et al. The association of race with childhood uveitis. Am J Ophthalmol. 2015;160(5):919-928.e1. doi:10.1016/j.ajo.2015.08.002

26. Acharya NR, Tham VM, Esterberg E, et al. Incidence and prevalence of uveitis: results from the pacific ocular inflammation study. JAMA Ophthalmol. 2013;131(11):1405-1412. doi:10.1001/ jamaophthalmol.2013.4237

27. Chauhan K, Rosenbaum J. Effect of age, race, sex and state of residence on ocular inflammatory and infectious diseases in the United States. Invest Ophthalmol Vis Sci. 2020;61(7):2056.

28. Yao S, Hong CC, Ruiz-Narváez EA, et al. Genetic ancestry and population differences in levels of inflammatory cytokines in women: role for evolutionary selection and environmental factors. PLoS Genet. 2018;14(6):e1007368. doi:10.1371/journal. pgen. 1007368

29. Wen X, Hu X, Miao L, et al. Epigenetics, microbiota, and intraocular inflammation: new paradigms of immune regulation in the eye. Prog Retin Eye Res. 2018;64:84-95. doi:10.1016/j.preteyeres.2018.01.001

30. Esen F, Alhan O, Kuru P, Sahin O. Safety assessment and power analyses in published anti-vascular endothelial growth factor randomized controlled trials. Am J Ophthalmol. 2016;169:68-72. doi:10.1016/j.ajo.2016.06.019
Clinical Ophthalmology

\section{Publish your work in this journal}

Clinical Ophthalmology is an international, peer-reviewed journal covering all subspecialties within ophthalmology. Key topics include: Optometry; Visual science; Pharmacology and drug therapy in eye diseases; Basic Sciences; Primary and Secondary eye care; Patient Safety and Quality of Care Improvements. This journal is indexed on PubMed

\section{Dovepress}

Central and CAS, and is the official journal of The Society of Clinical Ophthalmology (SCO). The manuscript management system is completely online and includes a very quick and fair peer-review system, which is all easy to use. Visit http://www.dovepress.com/ testimonials.php to read real quotes from published authors 